\title{
Research on a Novel Robust Disturbance Observer for an Uncertain Nonlinear System
}

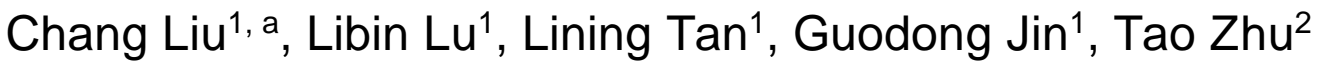 \\ ${ }^{1}$ Xi'an Research Institute of High Technology, Xi'an 710025, China \\ ${ }^{2}$ The Xi'an Surveying and Mapping Technological Center, Xi'an 710054, China \\ a2994353099@qq.com
}

\begin{abstract}
Keywords: nonlinear system, uncertainty, sliding mode, disturbance observer.
\end{abstract}
\begin{abstract}
A new robust disturbance observer was proposed in the article in order to estimate the modelling uncertainty and external disturbances. Based on the design of the nonlinear disturbance observer, in order to enhance its robustness, the observer is improved by the application of the principle of sliding mode. Then the proof on the stability of the observer and the principle of the parameter design are given. The simulation results reveal that the proposed method has higher estimation precision, speed, and stronger robustness compared with the above two kinds of observer.
\end{abstract}

\section{Introduction}

In order to effectively solve the problem of control of nonlinear uncertain systems subject to external disturbances, the researchers have proposed a number of methods. The method in which the disturbance observer is used to estimate the external disturbances and model uncertainties, and then to compensate for the interference Methods are increasingly valued and favored by researchers.

In [1], the concept of the extended disturbance observer is proposed for the first time, but it designed the disturbance observer is for linear system and lacks consideration for nonlinear system. In the literature [2 3], the design of the observer is further considered. A nonlinear extended disturbance observer is designed based on the design idea of the nonlinear disturbance observer and the extended disturbance observer,simulation experiments verify the effectiveness of the observer.

On the other hand, the introduction of the principle of sliding mode also provides a new idea for the design of disturbance observer. In [4], a sliding mode disturbance observer is designed based on the super-helix algorithm to estimate uncertain factors and the simulation results verify its effectiveness. In order to further improve the accuracy of the estimated interference, a high-order sliding mode disturbance observer is designed and applied to the aircraft control and navigation system based on the existence and bounded assumption of the higher order derivative of the disturbance in [5 7].

In this paper, we first study and analyze the design method and idea of the nonlinear disturbance observer and the extended disturbance observer. On this basis, the sliding mode principle is introduced to design a sliding mode extended disturbance observer, and its design principle,stability proof and simulation comparison verification is given.

\section{Design of a Novel Robust Disturbance Observer}

Based on the design method of nonlinear expansion disturbance observer and the principle of sliding mode, a design method of sliding mode extended disturbance observer is studied to further enhance the robustness of the observer.

Considering the following uncertain nonlinear system equations:

$$
\dot{x}=f(x)+g(x) u+d(t, x, u)
$$

where $d(t, x, u)$ represents the system uncertainty, parameter disturbance and external disturbance.

Suppose $d(t)$ is bounded and there is a constant $\mu>0$, making 


$$
\left|\frac{d^{i} d(t)}{d t^{i}}\right| \leq m \quad i=0,1,2
$$

Then the designed disturbance observer is:

$$
\left\{\begin{array}{l}
\dot{z}=f(x)+g(x) u+\hat{d} \\
\hat{d}=z_{1}+p_{1}(x) \\
\dot{z}_{1}=-L_{1}(x)(f(x)+g(x) u+\hat{d})+l_{1} \operatorname{sat}(s)+\hat{d} \\
\hat{\dot{d}}=z_{2}+p_{2}(x) \\
\dot{z}_{2}=-L_{2}(x)(f(x)+g(x) u+\hat{d})+l_{2} \operatorname{sat}(s)
\end{array}\right.
$$

Where $p_{i}(x), i=1,2$ is the function vector to be designed, $L_{i}(x)=\frac{\partial p_{i}(x)}{\partial x}, i=1,2$. Adjustable parameter $l_{i}$ uses a bandwidth-based configuration method that can change the convergence rate or bandwidth of the observer [3].

$$
\text { Set } e=\left[\begin{array}{c}
\tilde{x} \\
\tilde{d} \\
\tilde{\dot{d}}
\end{array}\right]=\left[\begin{array}{c}
x-z \\
d-\hat{d} \\
\dot{d}-\hat{\dot{d}}
\end{array}\right] \text {, and }\left\{\begin{array}{l}
\dot{\tilde{x}}=\dot{x}-\dot{z}=d-\hat{d} \\
\dot{\tilde{d}}=\dot{d}-\dot{\hat{d}}=\tilde{\dot{d}}-L_{1}(x) \tilde{d}-l_{1} \operatorname{sat}(\tilde{x}) \\
\tilde{d}^{(2)}=d^{(2)}-\hat{d}^{(2)}=d^{(2)}-L_{2}(x) \tilde{d}-l_{2} \operatorname{sat}(\tilde{x})
\end{array}\right.
$$

So

$$
\dot{e}=\left[\begin{array}{ccc}
0 & 1 & 0 \\
0 & L_{1}(x) & 1 \\
0 & L_{2}(x) & 0
\end{array}\right] e+\left[\begin{array}{l}
0 \\
0 \\
1
\end{array}\right] d^{(2)}-\left[\begin{array}{lll}
0 & & \\
& l_{1} & \\
& & l_{2}
\end{array}\right]\left[\begin{array}{l}
0 \\
\operatorname{sat}(\tilde{x}) \\
\operatorname{sat}(\tilde{x})
\end{array}\right]
$$

Which is $\dot{e}=A e+B d^{(r+1)}+L v \cdot(|\operatorname{sat}(\tilde{x})| \leq \alpha)$

For any given symmetric positive definite matrix $Q$, a symmetric positive definite matrix $P$ can always be found to make $A^{T} P+P A=-Q$.

Taking the Lyapunov function $V=e^{T} P e$, Its derivative is as follow:

$$
\begin{aligned}
& \dot{V}=\dot{e}^{T} P e+e^{T} P \dot{e} \\
& =\left(e^{T} A^{T}+\ddot{d}^{T} B^{T}-v^{T} L^{T}\right) P e+e^{T} P(A e+B \ddot{d}-L v) \\
& =e^{T}\left(A^{T} P+P A\right) e+\left(\ddot{d}^{T} B^{T} P e+e^{T} P B \ddot{d}\right)-\left(v^{T} L^{T} P e+e^{T} P L v\right) \\
& =-e^{T} Q e+2 e^{T} P B \ddot{d}-2 e^{T} P L v \\
& \begin{cases}=0 & e=0 \\
\leq 2\|e\|(\|P B\| m-\|P L\| \alpha)-\lambda_{m}\|e\|^{2} & e \neq 0\end{cases}
\end{aligned}
$$

Where $\lambda_{m}$ is the Minimum eigenvalue of $Q$. L is designed to make $(\|P B\| \mu-\|P L\| \alpha)<0$, the norm of the observed error vector $e$ will gradually converge to zero.

\section{Simulation}

In order to verify the effectiveness and superiority of the designed sliding mode extended disturbance observer, consider the following second order nominal system equation:

$$
\left[\begin{array}{l}
\dot{x}_{1} \\
\dot{x}_{2}
\end{array}\right]=\left[\begin{array}{cc}
0 & 1 \\
0 & -b_{n}
\end{array}\right]\left[\begin{array}{l}
x_{1} \\
x_{2}
\end{array}\right]+\left[\begin{array}{l}
0 \\
a_{n}
\end{array}\right] u+\left[\begin{array}{l}
0 \\
1
\end{array}\right] f_{e}
$$


where $f_{e}=d+\Delta+\left(b-b_{n}\right) x+\left(a-a_{n}\right) u, d$ is external disturbance, $\Delta$ is unmodeled nonlinear dynamic parts. Nominal system parameters are: $b_{n}=100, a_{n}=1$.

Setting $p_{1}(x)=m_{1} x, p_{2}(x)=m_{2} x$ in the DOB, the order of the observer is 2 order, then the observer is:

$$
\left\{\begin{array}{l}
\dot{z}=f(x)+g(x) u+\hat{d} \\
\hat{d}=z_{1}+m_{1} x \\
\dot{z}_{1}=-m_{1}(f(x)+g(x) u+\hat{d})+l_{1} \operatorname{sat}(s)+\hat{\dot{d}} \\
\dot{\dot{d}}=z_{2}+m_{2} x \\
\dot{z}_{2}=-m_{2}(f(x)+g(x) u+\hat{d})+l_{2} \operatorname{sat}(s)
\end{array}\right.
$$

This article takes $m_{1}=m_{2}=l_{1}=l_{2}=100, k_{p}=3, k_{i}=1, k_{d}=0.5$.The parameters in NDOB and EDOB are both 100 .

Calculate the "distance" between the estimated value of the disturbance and the true value according to the formula (14) as an index to evaluate the effect of the disturbance estimation.

$$
d_{l}=\sqrt{\sum\left(d_{-} \text {truth }-d_{-} \text {estimate }\right)^{2}}
$$

Assuming that the external disturbance is a fast time-varying disturbance consisting of square wave, sine wave and white noise and the rest is zero. The simulation results are as follows:

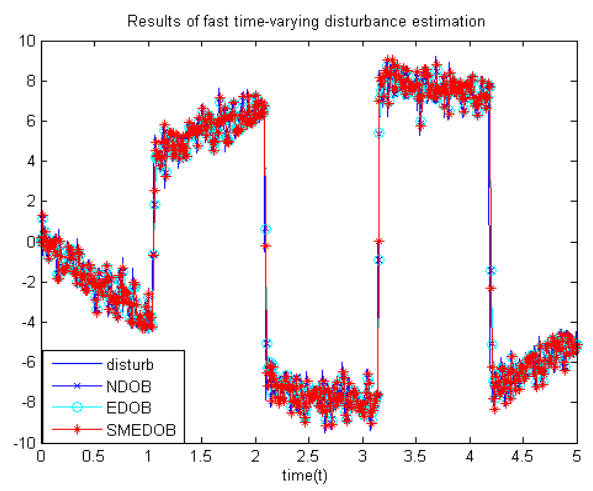

Fig 1(a) Results of fast time-varying disturbance estimation

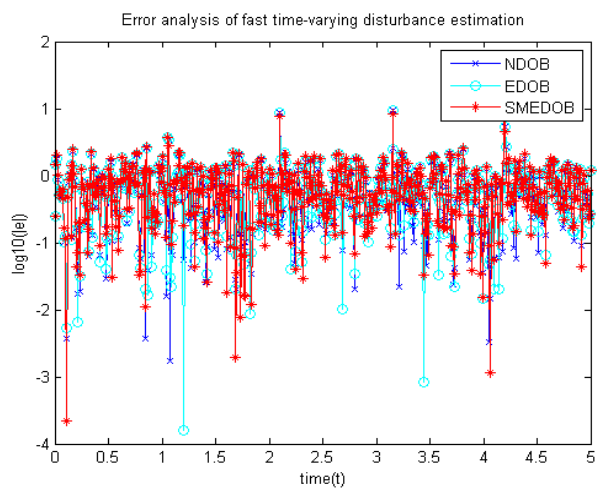

Fig 1(b) Error analysis of fast timevarying disturbance estimation

It can be seen from Fig. 1 (a) that the convergence rate of the new DOB is the fastest when the disturbance is abrupt, and the data in Table 1 shows that its observation accuracy is slightly higher than that of the other two observers.

Assuming model uncertainty, etc. Include:

parameter disturbance is $a=-102+40 \sin ^{2}(x)$ and unmodeled nonlinear dynamics is $\Delta=x_{2}^{2} \sin \left(x_{1}\right)+x_{2}$

In the case of external interference is zero, the simulation results are as follows: 


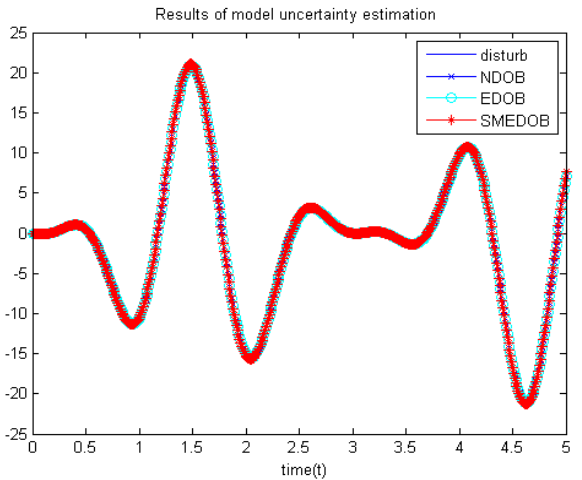

Fig 2(a) Results of model uncertainty estimation

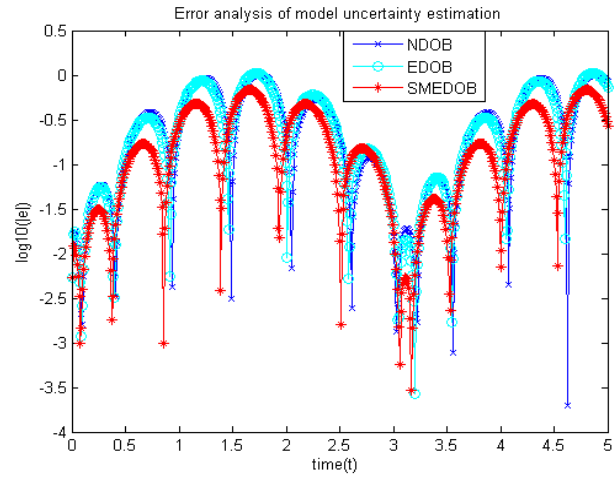

Fig 2(b) Error analysis of model uncertainty estimation

It can be seen from the corresponding data in Fig. 2 and Table 1 that the performance of the new DOB in estimating the disturbance caused by model uncertainty is significantly better than that of the other two observers due to the fact that the sliding mode control uncertainty is inherently robust.

Assuming that the interference is a set of disturbance with external fast time-varying disturbance and model uncertainty, the simulation results are as follows:

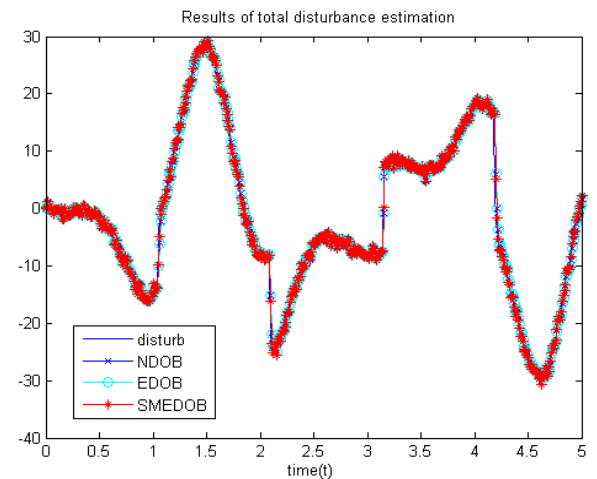

Fig 3(a) Results of total disturbance estimation

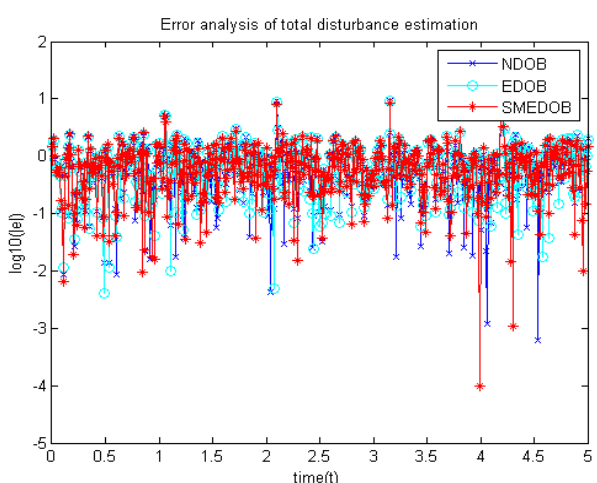

Fig3(b) Error analysis of total disturbance estimation

It can be seen from Fig. 3 (a) to (b) that the new DOB is more robust to the measurement noise than the other two observers.

Table 1 Disturbance effect evaluation data

\begin{tabular}{cccc}
\hline & NDOB & EDOB & SMEDOB \\
\hline fast time-varying disturbance & 28.0044 & 28.0539 & 27.8369 \\
model uncertainty & 10.6946 & 10.5564 & 6.6218 \\
total disturbance & 31.5069 & 31.4861 & 29.6219 \\
\hline
\end{tabular}

\section{Conclusion}

In this paper, we study the observer structure and disturbance estimation problem for an uncertain nonlinear system. Based on the design idea of nonlinear disturbance observer and extended disturbance observer and combined with the principle of sliding mode, a novel robust disturbance observer is proposed, and its stability proof and parameter design principle are given. The design of the disturbance observer inherits the advantages of the extended disturbance observer, relaxes the limitation of the change rate of the composite disturbance, and enhances the robustness of the observer by using the sliding mode principle. The simulation results show that the observer designed in this paper has higher accuracy than the other two observers for time-varying non-matching uncertainties, especially for system model uncertainty and measurement noise. 


\section{References}

[1]. Divyesh Ginoya, P.D.Shendge, S.B.Phadke, "Sliding Mode Control for Mismatched Uncertain Systems Using an Extended Disturbance Observer", IEEE TRANSACTIONS ON INDUSTRIAL ELECTRONICS, 2014, 61 (4):1983- 1992.

[2]. Zhou Jun,Zhao Jin-Long. Based on the extended disturbance observer of reentry vehicle terminal sliding mode control[J].Journal of Northwestern Polytechnical University, 2016,34(5): 818-822.

[3]. Zhang Jiao, Yang Xu,Liu Yuan-Xiang. Based on the extended disturbance observer constraint guidance law with attack Angle[J]. Journal of Beijing University of Aeronautics and Astronautics, 015, 41(12):2256-2267.

[4]. Gong Qing-Kun. SMDO Based Super-Maneuverable Flight Control for Fighters[J].Electronics Optics \& Control, 2014, 21(11):14-23.

[5]. Levant A.Non-homogeneous finite-time-convergent differentiator[C]//Joint 48th IEEE Conference on Decision and Control and 28th Chinese Control Conference, Shanghai, China, December 16-18, 2009.

[6]. Li Peng. Research and Application of Traditional and Higher-Order Sliding Mode Control [D].Chang Sha: Graduate School of National University of Defense Technology, 2011: 20-36.

[7]. WU Kun,FAN Jinsuo, ZHANG Hexin,XIA Zhaohui.A New Disturbance Observer Design Based on Higher-order Sliding Mode Control [J]. Journal of Projectiles,Rockets,Missiles and Guidance,2012, 32(4):199-202 